\title{
Tensor MRI Regularization via Graph Diffusion
}

\author{
Fan Zhang and Edwin R. Hancock \\ Department of Computer Science, University of York, York, UK
}

\begin{abstract}
A new method for diffusion tensor (DT) image regularization is presented that relies on heat diffusion on discrete structures. We represent a DT image using a weighted undirected graph, where the weights of the edges are determined from the geometry of the white matter fiber pathways. Diffusion across this weighted graph is captured by the heat equation, and the solution, i.e. the heat kernel, is found by exponentiating the Laplacian eigen-system with time. DT image regularization is accomplished by convolving the heat kernel with each component of the diffusion tensors, and its numerical implementation is realized by using the Krylov subspace technique. The algorithm is tested and analyzed both on synthetic and real DT images.
\end{abstract}

\section{Introduction}

DT-MRI is an important tool for non-invasive exploration of the anatomic structure of white matter in vivo. At each voxel, the DT-MRI produces a $3 \times 3$ diffusion tenor which measures the anisotropic behavior of water diffusion in brain white matter. The diffusion tensor at each voxel is able to distinguish the principal orientation of diffusion, which corresponds to the dominant axis of the white matter fiber bundles traversing the voxel. However, due to the motion of the ventricles and the partial volume effect, DT images are often noisy. Thus, regularization is necessary before fiber tracking can commence.

Generally, DT image regularization can be realized in one of the three ways. These are a) directly smoothing the diffusion weighted images (DWIs) before computing the tensors $[11,8]$, b) separately smoothing the eigenvectors and eigenvalues of the tensors $[2,3,5]$, and c) smoothing whole tensors [1, 7]. In the first method, one borrows techniques from classical gray-scale image processing to smooth the DWIs before estimation of the diffusion tensors. For instance, Parker et al. [11] applied the Perona-Malik algorithm to perform nonlinear smoothing of the raw DWIs, while Vemuri et al. [8] used a smoothing scheme based on the weighted total variation norm. The main drawback of this first group of methods is that the DWIs are smoothed independently without any constraints imposed by the tensors. In the second group of methods, vector regularization methods are used to smooth the principal eigenvectors of the tensors, and this is followed by diffusivity restoration. These methods make use of the fact that the fiber orientations are aligned with the principal eigenvectors. Poupon et al. [2] used a Markovian model to regularize the principal directions according to the structure of the fiber bundles. Using the same framework, Coulon et al. [5] regularized the principal diffusion directions using the classical total variational model. Since this second class of methods only consider the principal eigenvectors and the corresponding eigenvalues, information conveyed by the tensors may lost. Moreover, due to the partial volume effect in DT images, the principal eigenvectors of voxels containing fiber crossings no longer coincide with the local orientations of the fibers. In the third class of methods, full diffusion tensor data are used for 
regularization. Due to the high dimensionality and the positive-definite constraint for tensor data, traditional image smoothing techniques are often inappropriate for DT images. The space of tensor data is no longer a linear one, but a curved convex half-cone. It is hence a Riemannian manifold with an affine-invariant metric [4, 7]. Chefd'Hotel et al. [1] regularized tensor fields using the flow of matrix-valued functions under orthogonal and spectral constraints. The numerical solution is implemented using geodesic marching on the manifold. Pennec et al. [7] developed a statistical framework for the tensor manifolds, and used this to generalize the operations of interpolation, Gaussian and anisotropic regularization for tensor fields. Previous methods falling into the third group did not consider information concerning the structure of the white matter. In addition, these approaches are often relatively complex and the computational demands can be high.

In this paper we present a new diffusion-based regularization method for DT images which combines the advantages of the second and third groups of algorithm discussed above. We drive the diffusion process along the white matter fiber pathways, and smooth the full tensor data. Unlike previous methods for image smoothing using continuous partial differential equations (PDEs), our method is based on heat diffusion on a discrete structure $[10,12]$. We treat a DT image as a purely discrete object - a weighted undirected graph which encodes the geometry of the whiter matter fiber pathways. The advantage of formulating the problem on a graph is that purely combinatorial operators that require no discretization may be used. We therefore incur no discretization errors. Diffusion across this weighted graph with time is captured by the heat equation, and the solution, i.e. the heat kernel, is found by exponentiating the Laplacian eigen-system with time. DT image regularization is accomplished by convolving the heat kernel with each component of the diffusion tensors. The positive-definiteness of the tensors is naturally preserved. The solution of the algorithm is implemented using the Krylov subspace projection techniques [15]. The proposed method is able to smooth out noise while preserving the structure of the fiber bundles.

The next section of this paper presents the preliminaries concerning DT images. Section 3 describes the new graph diffusion method in detail. Experimental results on synthetic and real data are shown in Section 4. Section 5 concludes the paper.

\section{Preliminaries}

\subsection{DT Images}

At each voxel, a diffusion tensor $D$ is represented by a $3 \times 3$ symmetric (semi)positivedefinite matrix, e.g. $D=\left(d_{i j}\right)_{i, j=1,2,3}$. Then, the diffusivity in any direction $\hat{v}$ is given by a quadratic form $\hat{v}^{T} D \hat{v}$. Let $\beta_{1} \geq \beta_{2} \geq \beta_{3} \geq 0$ be the eigenvalues of $D$, and let $\hat{\varepsilon}_{i}$ be the normalized eigenvector corresponding to $\beta_{i}$. The tensor $D$ can then be described by $D=\beta_{1} \hat{\varepsilon}_{1} \hat{\varepsilon}_{1}^{T}+\beta_{2} \hat{\varepsilon}_{2} \hat{\varepsilon}_{2}^{T}+\beta_{3} \hat{\varepsilon}_{3} \hat{\varepsilon}_{3}^{T}$. The first eigenvector $\hat{\varepsilon}_{1}$, called the principal direction of diffusion, plays the most important role in the eigensystem, since it coincides with the local orientation of fibers in the white matter. However, in gray matter or cerebrospinal fluid, the water diffusion is almost isotropic, so the $\hat{\varepsilon}_{1}$ field loses its coherence and becomes meaningless and has a random orientation. We can use fractional anisotropy (FA) [6] to evaluate the tissue microstructure at each voxel:

$$
F A(D)=\frac{1}{\sqrt{2}} \frac{\sqrt{\left(\beta_{1}-\beta_{2}\right)^{2}+\left(\beta_{2}-\beta_{3}\right)^{2}+\left(\beta_{1}-\beta_{3}\right)^{2}}}{\sqrt{\beta_{1}^{2}+\beta_{2}^{2}+\beta_{3}^{2}}} .
$$


FA takes on values between 0 (fully isotropic diffusion) and 1 (infinite anisotropy). Thus low values indicate media with no preferred orientation, such as gray matter, whereas high values indicate tissues with strong orientation.

\subsection{Space of Diffusion Tensors}

Let us denote the space of all diffusion tensors as $M$, which forms a convex half-cone in the vector space of $3 \times 3$ matrices. The differential geometry of $M$ has been used in $[1,4,7]$ for DT image smoothing, segmentation and interpolation. The space $M$ is a Riemannian symmetric manifold with an affine-invariant metric. Following [4, 7], we define the Riemannian metric at each point $P \in M$ as the positive-definite inner product $\langle X, Y\rangle_{P}=\operatorname{tr}\left(P^{-1} X P^{-1} Y\right), X, Y \in T_{P} M$, where $T_{P} M$ is the tangent space at $P$. Thus, for a smooth curve $C:[a, b] \rightarrow M$ in $M$, the length of $C(t)$ can be computed via this metric, i.e. $\ell(C)=\int_{a}^{b}\left\|C^{\prime}(t)\right\|_{C(t)}=\int_{a}^{b} \sqrt{\operatorname{tr}\left(C(t)^{-1} C^{\prime}(t)\right)^{2}}$. As discussed in [4], the geodesic with initial point at identity matrix $I$ and tangent vector $X \in T_{I} M$ is given by $\exp (t X)$. Using the group action, an arbitrary geodesic $\Gamma(t)$ such that $\Gamma(0)=P$ and $\Gamma^{\prime}(0)=X$ is given by $\Gamma_{(P, X)}(t)=P^{\frac{1}{2}} \exp \left(t P^{-\frac{1}{2}} X P^{-\frac{1}{2}}\right) P^{\frac{1}{2}}$. Thus, the geodesic distance between two points $A$ and $B$ in $M$ is

$$
d_{g}(A, B)=\left\|\log \left(A^{-1} B\right)\right\|_{F}=\sqrt{\sum_{i=1}^{n}\left(\log \delta_{i}\right)^{2}},
$$

where $\delta_{i}$ are the eigenvalues of $A^{-1} B$. The quantity $d_{g}(A, B)$ is a natural measurement of the similarity between the two diffusion tensors $A$ and $B$, and may be used instead of the Euclidean distance between them in the vector space of matrices.

\section{Method}

The algorithm described in this paper is motivated by heat diffusion, which is one of the main techniques in image processing since the pioneering work of Perona and Malik [14]. We formulate the DT image smoothing through heat diffusion on graphs, which provides us a simple and elegant method to solve the problem. We describe the algorithm in three stages. These are a) generating the graph weights, b) establishing and solving the system of diffusion equations, and c) practical details of implementation.

To commence, we represent a DT image volume using a weighted undirected graph $G=(V, E)$, where the nodes (vertices) $V$ of the graph are the voxels of the image, and an edge is formed between every pair of nodes, i.e. two nodes $v_{i}$ and $v_{j}$ are connected by an edge $e_{i j} \in E$. The weight of an edge, $e_{i j}$, is denoted by $w\left(e_{i j}\right)$ or simply $w_{i j}$. The edge weights play an important role in our graph diffusion method, since they control the flow of heat across the graph. If the edge weight $w_{i j}$ is large, then heat can flow easily between nodes $v_{i}$ and $v_{j}$. By contrast, if $w_{i j}$ is small, it is difficult for heat to flow from $v_{i}$ to $v_{j}$.

\subsection{Edge Weights}

In order to represent the image structure by a graph without losing information, we must define a function that maps changes in the image data to edge weights. This is a common feature of all graph-based algorithms for image analysis [17, 13]. Amongst them, the Gaussian weighting function is the most popular one used to characterize the relationship between different pixels (voxels). In order to apply the Gaussian weighting function to DT images, we measure the similarity of two diffusion tensors using the geodesic distance 
between them (instead of the Euclidean distance used by the scalar and vector images). Thus, we compute the edge weight of $e_{i j}$ by

$$
w_{i j}=\exp \left(-\frac{d_{g}^{2}\left(D_{i}, D_{j}\right)}{\sigma^{2}}\right),
$$

where $D_{i}$ and $D_{j}$ are the diffusion tensors at voxels $i$ and $j$ respectively and $d_{g}$ is the geodesic distance given in Equation (2). In adapt the choice of $\sigma$ to images of different contrast, we normalize the distances $d_{g}$ before application of (3).

Although the above weighting method works well for traditional MRI volumes and gray-scale images whose image intensities are piecewise constant, it is not useful for distinguishing the details in brain white matter in DT images, where a large number of thin and long fibers may cross each other. In order to encode the geometry of the fiber bundles into our graph representation, we calculate the edge weight in white matter using the principal directions of the diffusion tensors [2]. These directions are consistent with the local orientations of the fibers. Thus, for each edge $e_{i j}$ connecting voxels $i$ and $j$ in white matter, we compute the angle $\theta(i, j)=\left(\hat{\varepsilon}_{1}\left(D_{i}\right), \hat{\varepsilon}_{1}\left(D_{j}\right)\right)$ where $\hat{\varepsilon}_{1}\left(D_{i}\right)$ denotes the first eigenvector of $D_{i}$. When $\theta(i, j)$ is an obtuse angle, we choose its supplementary angle. If voxels $i$ and $j$ belong to the same bundle, the angle $\theta(i, j)$ is small. Moreover, we can determine whether a voxel is in gray matter or white matter by evaluating the value of the fractional anisotropy. Therefore, for an edge $e_{i j}$ in white matter, we compute the edge weight using

$$
w_{i j}=\exp \left(-\frac{\left(\cos ^{-1}\left(\hat{\varepsilon}_{1}\left(D_{i}\right) \cdot \hat{\varepsilon}_{1}\left(D_{j}\right)\right)\right)^{2}}{\sigma^{2}}\right), \text { if } \operatorname{FA}\left(D_{i}\right), \operatorname{FA}\left(D_{j}\right) \geq \kappa,
$$

where $\operatorname{FA}(\cdot)$ is defined in Equation (1) and $\kappa$ is typically set to $0.1 \sim 0.2$ (as in fiber tracking algorithms [16]). Also, we normalize the angles $\theta(i, j)$ before application of (4). We have evaluated both the weighting functions (3) and (4) in our experiments, and (4) works better for DT images. However, (4) is only for DT images, while (3) can be used to all tensor-valued fields without preferred structures (as DT images have).

\subsection{Graph Diffusion}

Since we want to adopt a graph-spectral approach we introduce the weighted adjacency matrix $W$ for the graph $G$ whose elements $W(i, j)$ are $w_{i j}$ if $e_{i j} \in E$, and 0 otherwise. We also construct the diagonal degree matrix $T$ with entries $T(i, i)=\operatorname{deg}(i)=\sum_{j \in V} w(i, j)$. From the degree matrix and the weighted adjacency matrix we construct the combinatorial Laplacian matrix $L=T-W$, whose elements are

$$
L(i, j)= \begin{cases}T(i, i)-w_{i i}, & \text { if } i=j, \\ -w_{i j}, & \text { if } v_{i} \text { and } v_{j} \text { are adjacent nodes } \\ 0, & \text { otherwise. }\end{cases}
$$

The spectral decomposition of the Laplacian is $L=\Phi \Lambda \Phi^{T}$, where $\Lambda=\operatorname{diag}\left(\lambda_{1}, \lambda_{2}, \ldots, \lambda_{|V|}\right)$ is the diagonal matrix with the eigenvalues ordered according to increasing magnitude $\left(0=\lambda_{1}<\lambda_{2} \leq \lambda_{3} \ldots\right)$ as elements and $\Phi=\left(\phi_{1}\left|\phi_{2}\right| \ldots \mid \phi_{|V|}\right)$ is the matrix with the correspondingly ordered eigenvectors as columns. Since $L$ is symmetric and positive semidefinite, the eigenvalues of the Laplacian are all positive. The eigenvector $\phi_{2}$ associated with the smallest non-zero eigenvalue $\lambda_{2}$ is referred to as the Fiedler-vector. 
With these definitions in place, we introduce the heat equation on graphs associated with the Laplacian [10], i.e.,

$$
\frac{\partial h_{t}}{\partial t}=-L h_{t}
$$

where $h_{t}$ is the heat kernel and $t$ is time. The heat kernel satisfies the initial condition $h_{0}=I_{|V|}$ where $I_{|V|}$ is the $|V| \times|V|$ identity matrix. The heat kernel can hence be viewed as describing the flow of heat across the edges of the graph with time. The rate of flow is determined by the Laplacian of the graph. The solution to the heat equation is found by exponentiating the Laplacian eigen-spectrum, i.e.

$$
h_{t}=e^{-t L}=\Phi e^{-t \Lambda} \Phi^{T} .
$$

The heat kernel is a $|V| \times|V|$ symmetric matrix, and for the nodes $i$ and $j$ of the graph $G$ the resulting element is $h_{t}(i, j)=\sum_{i=1}^{|V|} e^{-\lambda_{i} t} \phi_{i}(i) \phi_{i}(j)$. When $t$ tends to zero, then $h_{t} \simeq$ $I-L t$, i.e. the kernel depends on the local connectivity structure or topology of the graph. If, on the other hand, $t$ is large, then $h_{t} \simeq e^{-t \lambda_{2}} \phi_{2} \phi_{2}^{T}$, where $\lambda_{2}$ is the smallest non-zero eigenvalue and $\phi_{2}$ is the associated eigenvector, i.e. the Fiedler vector. Hence, the large time behavior is governed by the global structure of the graph.

The hear kernel $h_{t}(i, j)$ decays exponentially with the weight $w_{i j}$ of the edge $e_{i j}$. It is useful to consider the following picture of the heat diffusion process on graphs. Suppose we inject a unit amount of heat at the node $m$ of a graph, and allow the heat to diffuse through the edges of the graph. The rate of diffusion along the edge $e_{i j}$ is determined by the edge weight $w(i, j)$. At time $t$, the value of the heat kernel $h_{t}(m, j)$ can be interpreted as the amount of heat accumulated at node $j$.

In order to use the diffusion to smooth a scalar image volume, we inject at each node an amount of heat energy equal to the intensity of the associated voxel (pixel). The heat at each node diffuses through the graph edges as time $t$ progresses. The edge weight plays the role of thermal conductivity. This heat diffusion process is governed by the same differential equation as (6), however, the initial conditions are different. If we encode the intensities of the image as a column vector $\rho$, then the evolution of the image intensity $\rho$ follows the equation

$$
\left\{\begin{array}{l}
\frac{\partial u_{t}}{\partial t}=-L u_{t} \\
u_{0}=\rho
\end{array}\right.
$$

and the solution is

$$
u_{t}=e^{-t L} \rho=h_{t} \rho .
$$

As a result the smoothed image intensity of voxel $j$ at time $t$ is $u_{t}(j)=\sum_{i=1}^{|V|} \rho(i) h_{t}(i, j)$. This is a measure of the total intensity to flow from the remaining nodes to node $j$ during the elapsed time $t$.

Since DT images are multidimensional, we let each component of the tensors diffuse on the graph constructed from the weighting function (4). The values of the image data in white matter can flow easily along the fiber bundles, while it is difficult for image data to flow from one bundle to another. In order to regularize DT images, we thus apply Equation (8) on each of the six independent components of the DT images, and this forms a system of six coupled PDEs. The coupling results from the fact that the edge weight (diffusivity) depends on all the image channels. As a result the smoothed diffusion tensor 
of voxel $j$ at time $t$ is

$$
u_{t}(j)=\sum_{i=1}^{|V|} h_{t}(i, j) D_{i} .
$$

Since each row $i$ of the heat kernel $h_{t}$ satisfies the conditions $0 \leq h_{t}(i, j) \leq 1$ for $\forall j$ and $\sum_{j=1}^{|V|} h_{t}(i, j)=1$, the smoothed result in (10) not only preserves the total tensor value over the set of voxels in the DT image, but also preserves the (semi)positive-definiteness of the diffusion tensors as proved in the following.

Proof of Positive-definiteness Conservation: Note that a diffusion tensor $D$ is semipositive definite if $\mathbf{x}^{T} D \mathbf{x} \geq 0$ for $\forall \mathbf{x} \in R^{3}$. Then, for each smoothed tenor at voxel $j$, we have $\mathbf{x}^{T} u_{t}(j) \mathbf{x}=\mathbf{x}^{T}\left(\sum_{i=1}^{|V|} h_{t}(i, j) D_{i}\right) \mathbf{x}=\sum_{i=1}^{|V|} h_{t}(i, j) \mathbf{x}^{T} D_{i} \mathbf{x} \geq 0$ due to the fact $h_{t}(i, j) \geq 0$. This proves the statement.

\subsection{Numerical Implementation}

A direct method to compute the smoothed images from (9) is via the heat kernel $h_{t}$, which can be found by spectral decomposition of the sparse matrix $L$, e.g. $h_{t}=\Phi e^{-t \Lambda} \Phi^{T}$, or matrix exponential, e.g. $h_{t}=e^{-t L}=I-t L+\frac{t^{2}}{2 !} L^{2}-\frac{t^{3}}{3 !} L^{3}+\cdots$. Unfortunately, current DT image volumes often exceed $128 \times 128 \times 64 \simeq 1 \times 10^{6}$ voxels, thus, most available computers do not have the ability to calculate all the eigenvalues and eigenvectors of such a large matrix, even using the well-known Lanczos algorithm which is often used to find the extremal eigenvalues of large sparse matrices. It is also impossible to calculate the polynomial series defined by the matrix exponential due to the uncertainty of the terms and computation complexity of the matrix-matrix multiplication.

As an alternative to find the heat kernel, the Krylov subspace projection technique [15, 9] allow us to compute the action of a matrix exponential operator on an operand vector, e.g. $e^{-t L} \rho$, without having to compute explicitly the matrix exponential operator in isolation. The underlying principal of the Krylov subspace technique is to approximate $e^{-t L} \rho$ by an element of the Krylov subspace spanned by the vectors $\left\{\rho,(-t L) \rho, \ldots,(-t L)^{m} \rho\right\}$ for some $m$ that is typically small compared to the dimension of $L$. The Krylov subspace technique is one of the iterative methods for sparse matrix problems, in which only matrix-vector multiplication is needed. These methods have the advantages of small memory requirement and the ability to represent the matrix-vector multiplication as a function (matrix-free method). For our'26-connected' $3 D$ lattices from DT images, the Laplacian matrix $L$ is very sparse with at most 26 circulant nonzero elements in each row and one may store the entire matrix as a vector of weights. Therefore, the operation of matrix-vector product may be performed very economically even for large $L$ if memory is at a premium. Moreover, Krylov subspace methods may be efficiently parallelized [9].

Obviously, solving the equation defined in (9) is the main computational bottleneck for the algorithm and requires the most time. The implementation of the Krylov subspace method for solving (9) in this paper relies on the Matlab subroutines from the Expokit package [15]. Tests on a PC with an Intel P4 2.4GHZ and 1.5GB memory show that it requires approximately 100, 75, 50, 25 seconds to solve (9) for the Laplacian matrices of '26-connected' graphs with $128 \times 128 \times 40,30,20,10$ nodes respectively.

\subsection{Algorithm}

To summarise, the steps of the algorithm are: 
1. Use the fractional anisotropy defined in (1) to choose the voxels of white matter and compute their principal directions.

2. Use (4) (If we choose the weight function (3), step one is not needed) to generate edge weights, $w_{i j}$, between "26-connected" nodes $v_{i}$ and $v_{j}$.

3. Solve (9) for each component of DT images using the Krylov subspace techniques.

\section{Experiments}

In this section, we present the experimental results of applying the proposed algorithm to both synthetic and real DT image volumes. Synthetic images of varying complexity were used to evaluate both qualitatively and quantitatively the effectiveness of the method. All our experiments use "26-connected" graphs.

There are two free parameters of our algorithm: 1) the width $\sigma$ of the Gaussian for edge weights and 2) the time $t$ used to terminate the diffusion. Due to the normalization of the distances for computing the edge weights, the algorithm performs in a stable way and obtains good results over a large range of values of these parameters. To demonstrate this, we generate a synthetic $8 \times 8 \times 6$ tensor field, and add the same quantity of independent and identically distributed (IID) additive noise into the eigenvalues and eigenvectors of the tensors, as shown in (a) and (b) of Fig. 2 respectively. We then apply our graph diffusion with weight function (4) to regularize the noisy tensor field using various values of $\sigma$ and $t$. To evaluate the regularized images, we use the root-mean-square error (RMSE) measure for the tensor field between a restored image and the corresponding noise-free original image. The RMSE is $R M S E=\sqrt{\sum_{i=1}^{i=N} d\left(D_{i}^{\prime}, D_{i}\right)^{2} / N}$, where $N$ is the number of voxels and $d\left(D_{i}^{\prime}, D_{i}\right)$ is the Euclidean distance, e.g. $d_{E}=\operatorname{tr}\left(\left(D_{i}^{\prime}-D_{i}\right)^{T}\left(D_{i}^{\prime}-D_{i}\right)\right)$ or Riemannian distance defined in (2) between two tensors. For each pair of values of $(\sigma, t)$, we compute the RMSE and plot the result in panel (c) of Fig. 2. The figure shows that the RMSE reaches its minimum values when $0.1 \leq \sigma \leq 0.25, t \geq 5$. The RMSE is insensitive to the value of $t$ if $\sigma$ is in this range. If $\sigma$ is too large (i.e. $\sigma>0.25$ in this case), then the image will be over-regularized, i.e. too smooth. In the other hand, $\sigma$ is too small $(<0.1$ in this case), then the data is smoothing negligible even when $t$ is very large. As a result noise can not be eliminated efficiently.

Fig. 3 shows the results of our graph diffusion on there synthetic noisy tensor fields. Field (a1) is fairly simple, while (a2) contains crossing fibers and (a3) mimics a fiber bundle. We regularize these images using both weight functions (4) and (3). The results of which are shown in column two and column three respectively. The diffusion based on weighting function (4) preserves well, and even restores, the fine details in the images while suppressing the noise. Although the weight function (3) can also work well in certain circumstances, it fails to restore the left edge of image (a2). Another problem for edge function (3), as shown in subfigure (c1), is that it may suffer from the eigenvalue swelling effect reported in [1]. The reason of this is as follows: When the noise does not affect the tensor eigenvectors (orientations) as much as the eigenvalues (diffusivities), the eigenvalues may contribute more than the eigenvectors for the edge weights computed using weight function (3). Thus, our global matrix regularization process may smooth the eigenvalues faster than the eigenvectors, resulting in an eigenvalue swelling effect for large-t regularization. On the other hand, edge weight function (4) only uses the main eigenvectors (which is the most important information for DT images). Thus, in this case the diffusion is dominated by the genuine structure of the white matter. For the 


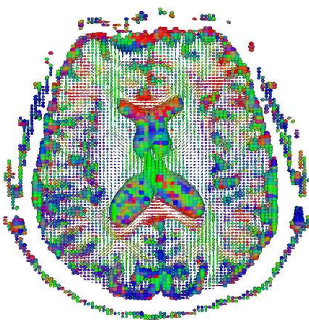

(a1)

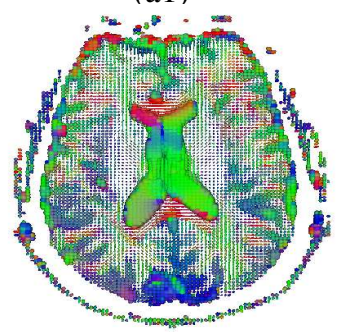

(a2)

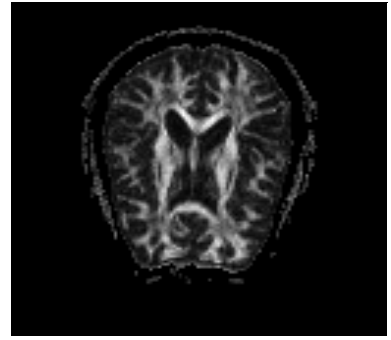

(b1)

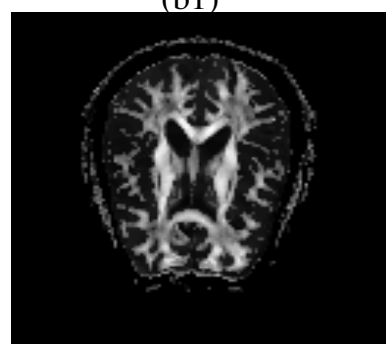

(b2)

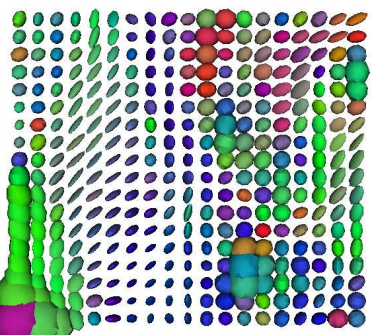

(c1)

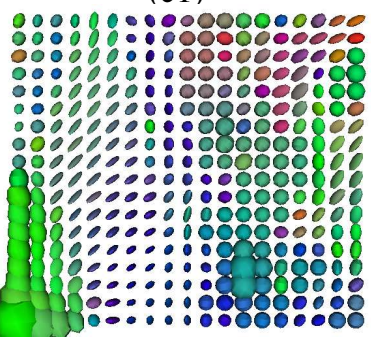

(c2)

Figure 1: (a1)(b1)(c1): A sample DTI slice, its FA map and a Region-Of-Interest from it. (a2)(b2)(c2): The corresponding regularized slice, FA map and ROI.

purposes of visual comparison with an alternative diffusion-based method, Fig. 3 shows the results of Riemannian anisotropic filtering (RAF) [7], which is computationally very time-consuming. The method performs rather badly on the noisy image (a2). Our experiments show empirically that RAF may reduce the length of the tensors (as shown in subfigure (d1)), especially when a large number of iterations are used.

In order to better analyze the behavior of our method, we also present quantitative comparisons of the proposed algorithm with RAF. We take the noise-free tensor field (a) in Fig.2 as the ground truth, and generate a sequence of noisy images with different levels of IID additive noise. Our method using both edge weight function (4) and (3) and RAF [7] were applied to the sequence of noisy images. To compare these methods, we computed the RMSE of the reconstructed images based on both Euclidean distance, which prefers our two methods, and the Riemannian distance, which prefers RAF. The results are plotted in Fig. 4. The plot shows that our method with weight function (4) gives the best result under both Euclidean and Riemannian RMSE, although the result of RAF is comparable with our method if we use Riemannian RMSE measurement.

We have applied our algorithm to a real-world DT-MR brain image of size $128 \times$ $128 \times 33$. We threshold the white matter voxels (131667 voxels in total in this case) from the FA map using a value of 0.15 , and use graph diffusion with weight function (4) to regularize these voxels. On the other hand, we use graph diffusion with weight function (3) to smooth the voxels (26490 voxels in total in this case) of the gray matter where $F A<0.15$. The entire process requires approximately 3.5 minutes using Matlab programming on the machine described before. Fig. 1 shows the results on a sample slice. The first row shows an ellipsoid representation, FA map and a Region-Of-Interest (ROI). The second row shows the smoothed counterparts. As shown by the smoothed FA map in subfigure (b2), discontinuities are well preserved and the local coherence of the anisotropy map is largely enhanced by the graph diffusion. The zoomed ROI in (c2) further validates 
the effectiveness of our method, which makes the fiber streamlines smoother while also smoothing out noise in the surrounding isotropic matter.

\section{Conclusion}

We have presented a new method for DT image regularization based on heat diffusion on graphs. We admit the discrete nature of images from the outset, and use graphs to represent DT images. Diffusion on these graphs is driven by the prior geometry of the whiter matter fiber bundles. The exact solution of the method is directly solved without numerical iteration as is the case with most PDE-based methods. This leads to simple and fast implementation. The algorithm smoothes the whole tensor while automatically ensuring the positive-definiteness constraint. Results on synthetic and real DT image data show that the method can preserve, and even enhance, the coherence of fiber structures while effectively smoothing out noise.

\section{References}

[1] C. Chefd'Hotel, D. Tschumperle, et al . Constrained flows of matrix-valued functions: Application to diffusion tensor regularization. In Proc. of ECCV, pages 251-265, 2002.

[2] C. Poupon, etc. Towards inference of human brain connectivity from mr diffusion tensor data. Medical Image Analysis, 5:1-15, 2001.

[3] D. Tschumperle and R. Deriche . Diffusion tensor regularization with constraints preservation. In Proceedings of CVPR(1) 2001, pages 948-953, 2001.

[4] M. Moakher . A differential geometric approach to the geometric mean of symmetric positivedefinite matrices. SIAM Journal on Matrix Analysis and Applications, 26(3):735-747, 2005.

[5] O. Coulon, D. Alexander, S. Arridge . Diffusion tensor magnetic resonance image regularization. Medical Image Analysis, 8:47-67, 2004.

[6] P.J. Baser and C. Pierpaoli . Microstructural and physiological features of tissues elucidated by quantitative-diffusion-tensor mri. J. of Magnetic Resonance, B, 111:209-219, 1996.

[7] X. Pennec, P. Fillard, N. Ayache . A riemannian framework for tensor computing. International Journal of Computer Vision, 66(1):41-66, 2006.

[8] B. Vemuri, Y. Chen, et al . Fiber tract mapping from diffusion tensor mri. In IEEE Workshop Variational and Level Set Methods in Computer Vision, pages 81-88, 2001.

[9] C. Moler, C. Van Loan. Nineteen dubious ways to compute the exponential of a matrix, twenty-five years later. SIAM Review, 45(1):3-49, 2003.

[10] F.R.K. Chung. Spectral Graph Theory. American Mathmatical Society, 1997.

[11] G. Parker, J. Schnabel, et al. Nonlinear smoothing for reduction of systematic and random errors in diffusion tensor imaging. J. of Magnetic Resonance Imaging, 11:702-710, 2000.

[12] R. Kondor and J. Lafferty. Diffusion kernels on graphs and other discrete structures. 19th Intl. Conf. on Machine Learning (ICML), 2002.

[13] L. Grady . Multilabel random walker image segmentation using prior models. In Proceedings of CVPR05, pages 763-770, 2005.

[14] P. Perona and J. Malik. Scale-space and edge dectection using anisotropic diffusion. IEEE Trans. Pattern Anal. and Machine Intell., 12(7):629-639, 1990.

[15] R. Sidje. Expokit: a software package for computing matrix exponentials. ACM Trans. on Mathematical Software, 24(1):130 - 156, 1998.

[16] S. Mori, P. Zijl. Fiber tracking - a technical review. NMR in Biomedicine, 15:468-80, 1998.

[17] J. Shi and J. Malik. Normalized cuts and image segmentation. TPAMI, 22(8):888-905, 2000. 


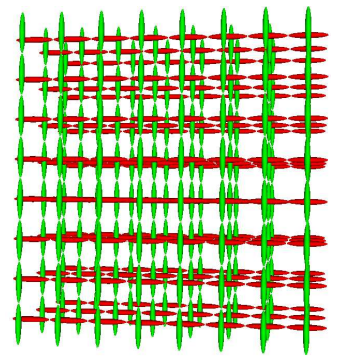

(a) Ground truth

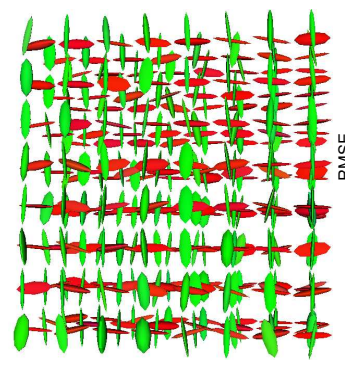

(b) Noisy image

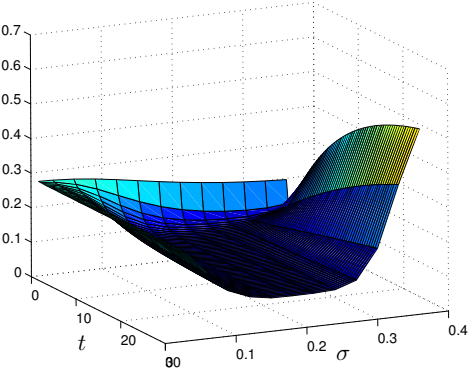

(c) Parameter stability

Figure 2: Parameter stability

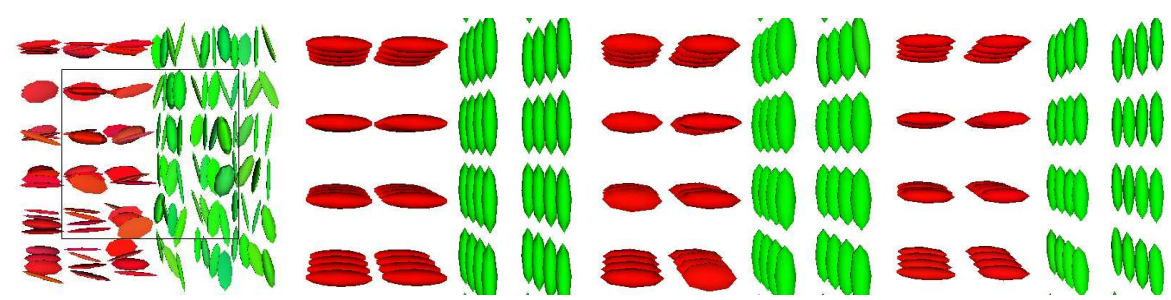

(a1)

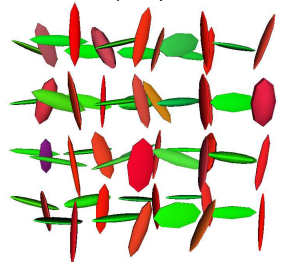

(a2)

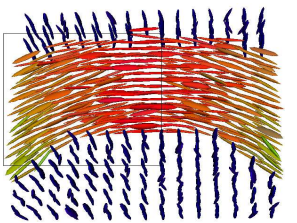

(a3) (b1)

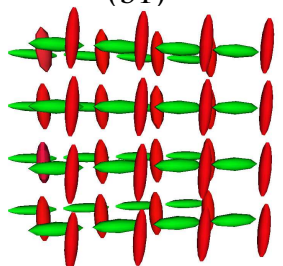

(b2)

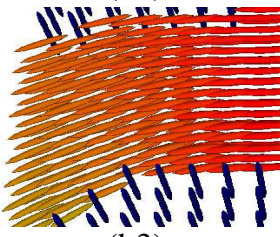

(b3) (c1)

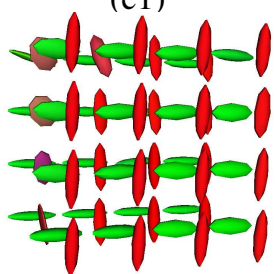

(c2)

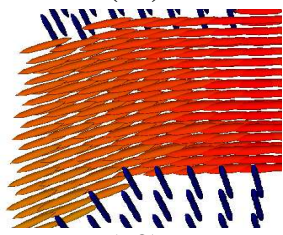

(c3) (d1)

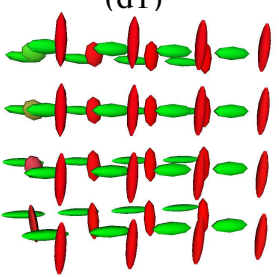

(d2)

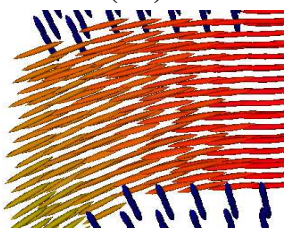

(d3)

Figure 3: (a1-3): Synthetic images. (b1-3): Graph method using (4) with $\sigma=0.15, t=7$. (c1-3): Graph method using (3) with $\sigma=0.15 t=7$. (d1-3): RAF [7] with 5 iterations.
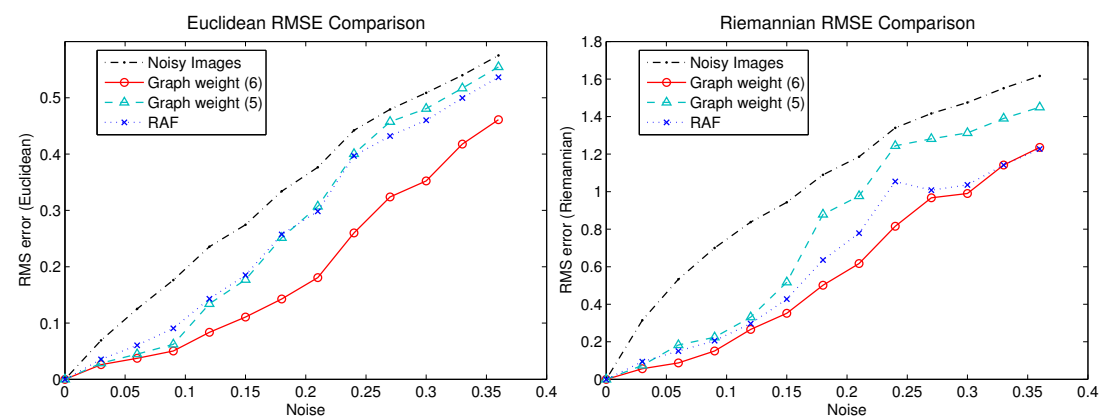

Figure 4: RMSE Comparison 